\title{
Photoacoustic endoscopic imaging study of melanoma tumor growth in a rat colorectum in vivo
}

Chiye Li, Joon-Mo Yang, Ruimin Chen, Yu Zhang, Younan Xia, et al.

Chiye Li, Joon-Mo Yang, Ruimin Chen, Yu Zhang, Younan Xia, Qifa Zhou, K. Kirk Shung, Lihong V. Wang, "Photoacoustic endoscopic imaging study of melanoma tumor growth in a rat colorectum in vivo," Proc. SPIE 8581, Photons Plus Ultrasound: Imaging and Sensing 2013, 85810D (4 March 2013); doi: $10.1117 / 12.2005470$

SPIE. Event: SPIE BiOS, 2013, San Francisco, California, United States 


\title{
Photoacoustic endoscopic imaging study of melanoma tumor growth in a rat colorectum in vivo
}

\author{
Chiye $\mathrm{Li}^{1,4}$, Joon-Mo Yang ${ }^{1,4}$, Ruimin Chen ${ }^{2}$, Yu Zhang ${ }^{3}$, Younan Xia ${ }^{3}$, Qifa Zhou ${ }^{2}$, K. Kirk \\ Shung $^{2}$, and Lihong V. Wang ${ }^{1 *}$ \\ ${ }^{1}$ Optical Imaging Laboratory, Department of Biomedical Engineering, Washington University in St. \\ Louis, One Brookings Drive, Campus Box 1097, St. Louis, Missouri, 63130, USA \\ ${ }^{2}$ Ultrasonic Transducer Resource Center, Department of Biomedical Engineering, University of \\ Southern California, 1042 Downey Way, University Park, DRB 130, Los Angeles, CA 90089, USA \\ ${ }^{3}$ The Wallace H. Coulter Department of Biomedical Engineering, Georgia Institute of Technology \\ and Emory University, Molecular Science and Engineering Building, 901 Atlantic Drive, Atlanta, \\ GA 30332, USA
}

\begin{abstract}
We performed a photoacoustic endoscopic imaging study of melanoma tumor growth in a nude rat in vivo. After inducing the tumor at the colorectal wall of the animal, we monitored the tumor development in situ by using a photoacoustic endoscopic system. This paper introduces our experimental method for tumor inoculation and presents imaging results showing the morphological changes of the blood vasculature near the tumor region according to the tumor progress. Our study could provide insights for future studies on tumor development in small animals.
\end{abstract}

Keywords: Photoacoustic endoscopy, melanoma tumor, nude rat, colorectum.

\section{INTRODUCTION}

Because of its strong spectroscopic and angiographic imaging ability, photoacoustic endoscopy (PAE) ${ }^{1-4}$ could be a powerful tool for studying tumor development. Importantly, PAE can provide plenty of functional information and blood vasculature information without using any contrast agent ${ }^{5-16}$. Although many in vivo photoacoustic (PA) imaging studies based on tumor models induced in mouse or rats have been reported ${ }^{8,17-21}$, to our knowledge, none of them were implemented in the endoscopic form.

In this study, we investigated such capability of PAE by performing a melanoma tumor imaging experiment with a nude rat. Like colonic carcinoma, melanoma tumor is a well-known epithelial tumor. By injecting the tumor cells (B16 melanoma cell line), we produced a xenograft melanoma tumor model at the colorectal wall of an athymic nude mutant rat (Hsd:RH-Foxn $1^{r m} /$ Foxn $1^{+}$). Then, we monitored its development in situ using a PA endoscopic system ${ }^{3}$ and could observe vasculature variation as the tumor developed. Here we describe the experimental methods for tumor induction and the endoscopic imaging procedure, and present the imaging results.

\section{MATERIALS AND METHODS}

\subsection{Photoacoustic endoscopic system}

\footnotetext{
${ }^{4}$ These authors contributed equally to this work. ${ }^{*}$ Corresponding author: 1hwang@biomed.wustl.edu
} 
For this study, we utilized the 3.8-mm diameter probe based endoscopic system reported in Nature Medicine ${ }^{3}$. Figure 1 shows the endoscope and its peripheral systems, composed of a micromotor driver circuit, a delay generator, a laser system, an ultrasonic (US) pulser-receiver including an amplifier, a data acquisition (DAQ) card, and a computer for recording signals and displaying images.

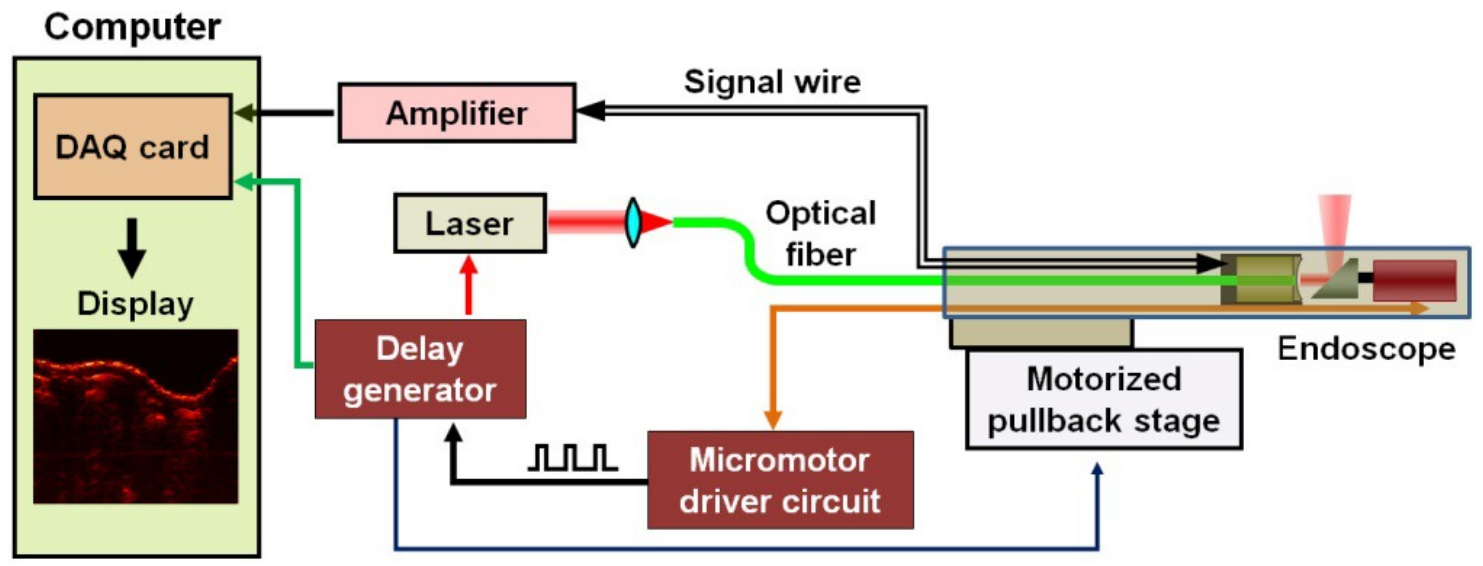

Figure 1. Block diagram showing the endoscopic probe and its peripheral systems.

For PA imaging, laser pulses ( $578 \mathrm{~nm}, \sim 10 \mathrm{~ns}$ pulse width, $\sim 0.3 \mathrm{~mJ} /$ pulse) from a tunable dye laser (Cobra HRR, Sirah), pumped by a solid-state, diode-pumped Nd:YLF laser (INNOSLAB IS811-E, EdgeWave), are guided by a multimode optical fiber (BFL22-365, Thorlabs) and emitted through the central hole of a single element focused US transducer $\left(\mathrm{LiNbO}_{3}, \sim 36 \mathrm{MHz}, 65 \%\right.$ fractional bandwidth), which is coaxially aligned with the optical fiber. After exiting the fiber, the laser beams are further directed to the target tissue by a scanning mirror, and finally generate PA waves once absorbed by the target tissue. The PA waves that propagate to the scanning mirror are reflected by the same mirror, sent to the US transducer, converted into electrical signals, amplified by the US pulser-receiver (5072PR, Panametrics), and digitally recorded by the DAQ card (NI PCI-5124, National Instruments). With the endoscopic system, we acquired volumetric PA images with a B-scan frame rate of $\sim 4 \mathrm{~Hz}$. More information on the endoscope's structure is available in our previous report ${ }^{3}$.

With the laser fluences used in these experiments, the endoscope's maximal radial imaging depth was $\sim 7 \mathrm{~mm}$ from the endoscope's surface, and the angular field-of-view (FOV) was limited to approximately $270^{\circ}$, due to partial blocking by the probe housing. Experimentally measured highest PA and US resolutions in the focal zone of the transducer were respectively $\sim 55 \mu \mathrm{m}$ and $\sim 30 \mu \mathrm{m}$ in the axial direction, and $\sim 80 \mu \mathrm{m}$ and $\sim 60 \mu \mathrm{m}$ in the transverse direction, but the transverse resolution varied with target distance.

\subsection{Tumor inducement}

Because of the long rigid distal section $(\sim 38 \mathrm{~mm})$ of the employed endoscope, we chose an athymic nude mutant rat (Hsd:RH-Foxn $1^{\text {rnu }} /$ Foxn $1^{+}$, Harlan Laboratories) for the host of melanoma tumor.

During the cell culture, we maintained B16 melanoma cells in Dulbecco's Modified Eagle Medium with 10\% fetal bovine serum and $1 \%$ penicillin/streptomycin supplement. The cells were incubated in $37 \mathrm{C}^{\circ}, 5 \% \mathrm{CO}_{2}$ and divided every 72 hours. For subculture, they were seeded at $2-4 \times 10^{4} \mathrm{cells} / \mathrm{cm}^{2}$ after being dispersed in $0.25 \%$ EDTA-trypsin. For injection, cells were resuspended in phosphate buffered saline after trypsinization. Then, we locally injected a 200$\mu 1$ tumor cell solution, containing $\sim 10^{6}$ tumor cells, to the colorectal wall of a nude rat ( $\sim 16$ weeks old) via anus by using a 30 gauge needle. To dilate the anus, we utilized a plastic catheter which had a small hole at the tip for the needle introduction. With this injection method, we were able to induce melanoma tumor while minimizing the anatomical disturbance of animal which frequently occurs in conventional surgical injection methods. 


\subsection{Endoscopic imaging of the tumor}

We targeted monitoring the tumor development with a one-week interval. When we appreciated that an endoscopic imaging experiment was needed, we fasted the rat for $\sim 20 \mathrm{hr}$ before the day of the experiment to increase the likelihood of an empty colon for endoscopic imaging. In each imaging experiment, we anesthetized the rat with $\sim 4 \%$ isoflurane for induction, and administered a cocktail of $87 \mathrm{mg} / \mathrm{kg}$ ketamine and $13 \mathrm{mg} / \mathrm{kg}$ xylazine (IP) to provide time to prepare and mount the animal. Once the animal was properly positioned, medical ultrasound gel was inserted into the colon via a small plastic tube. The ultrasound gel provided acoustic coupling between the tissue and US transducer and lubricated the probe during colon insertion through the anus. Then we inserted the endoscopic probe into the colon $\sim 6 \mathrm{~cm}$ deep from the animal's anus and performed pullback volumetric scans over a $\sim 2-3 \mathrm{~cm}$ range during constant pullback translation of the probe at a mechanically governed speed of $\sim 160 \mu \mathrm{m} / \mathrm{s}(\sim 1 \mathrm{~cm} / \mathrm{min})$. We recorded multiple volumetric data sets, and each volumetric scan required a scanning time of $\sim 2-3$ min. About 500-800 B-scan slices with longitudinal spacings of $\sim 40 \mu \mathrm{m}$ were acquired for each imaging session. While imaging, we maintained anesthesia with $1.5-2.0 \%$ isoflurane supplied through a nose cone and monitored its vital signs during the experiment. Once we observed that the tumor grew up too big to perform endoscopic imaging, we euthanized the animal by a pentobarbital overdose $(150 \mathrm{mg} / \mathrm{kg}$, IP) and surgically validated.

All procedures in the experiment followed the protocol approved by the Institutional Animal Care and Use Committee at Washington University in St. Louis.
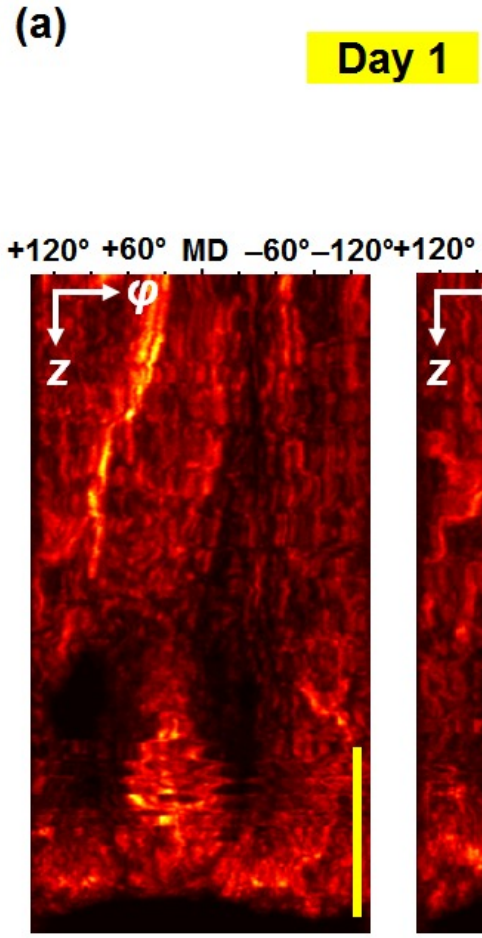

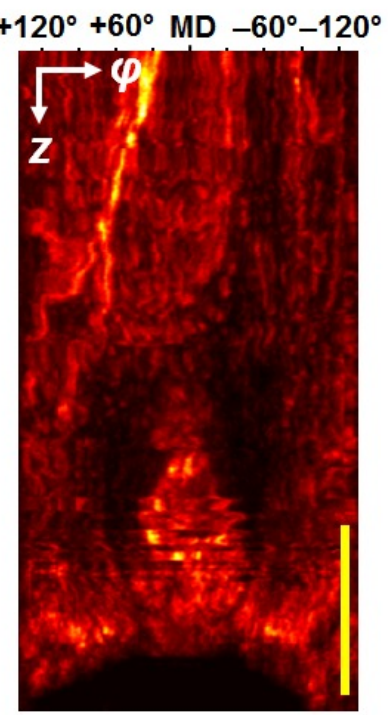

(b)

Day 2

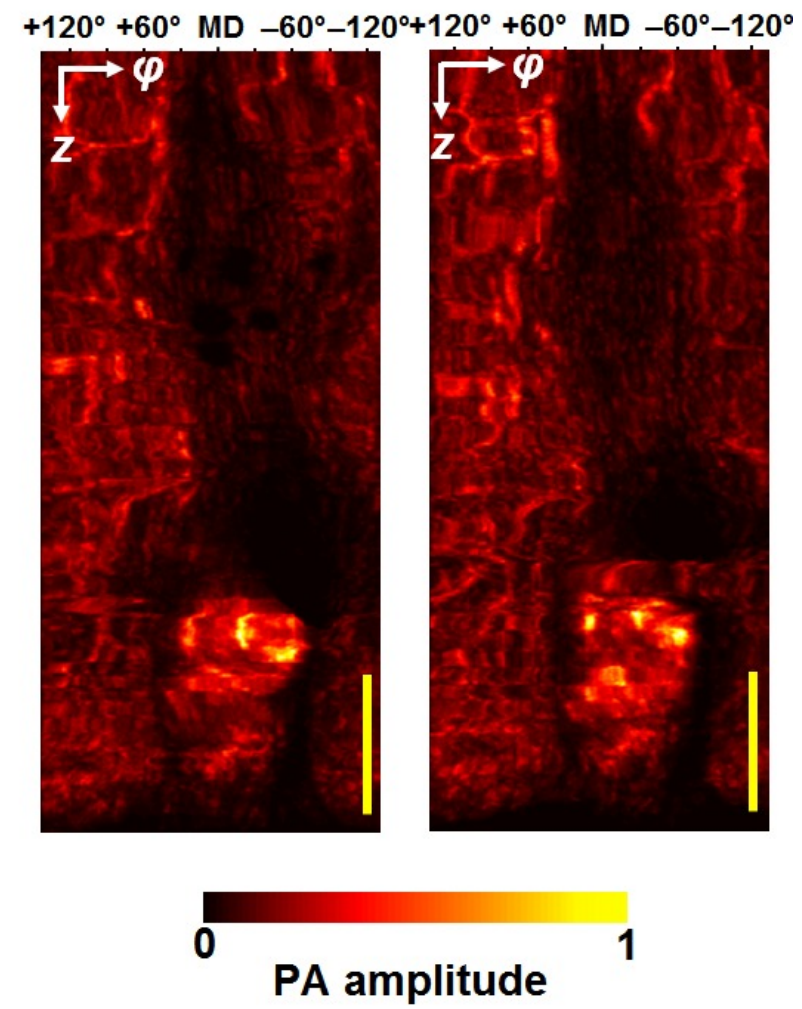

Figure 2. Serially-acquired PA-RMAP images from the rat colorectum with a melanoma tumor (views from the inside of the colon). In each image, the horizontal $\varphi$-axis corresponds to the angular FOV covering $270^{\circ}$, and the vertical $z$-axis corresponds to the pullback length of $2-3 \mathrm{~cm}$. The approximate mid-dorsal (MD) position and angular measures from the MD are marked along the horizontal $\varphi$-axis, where the positive and negative values correspond to the right and left sides of the animal. The scale bars represent $5 \mathrm{~mm}$ for the vertical direction only. 


\section{RESULTS AND DISCUSSION}

In general, tumor growth speed shows a large variation depending on the health condition of the animal and the environment of the tumor injection site. So, we frequently checked the tumor progress status and decided the starting moment of endoscopic imaging experiment. In this study, we performed the first endoscopic imaging after two weeks from the tumor cell injection, and could acquire endoscopic image data twice with a one-week interval.

In Figure 2, we present the serial PA radial-maximum amplitude projection (RMAP) images acquired from the rat using a $578 \mathrm{~nm}$ laser wavelength. To show the image reproducibility, we present two sets of image data in parallel for each experiment day. As shown in the images, the tumor region was clearly visualized in PA images because the melanoma tumor tissue includes a lot of highly light-absorbing melanin. Also, one can see the increase of the tumor size and adjacent vasculature change in the colorectal wall clearly between the first and second experiment days.

To show the melanoma tumor's configuration in a three-dimensional space, we produced a volume-rendered PA image using the data presented in Figure 2(b), and present it with a photograph image in Figure 3. As shown in Figure 3(a), the melanoma tumor location and its adjacent vasculature were clearly mapped by the PA imaging.
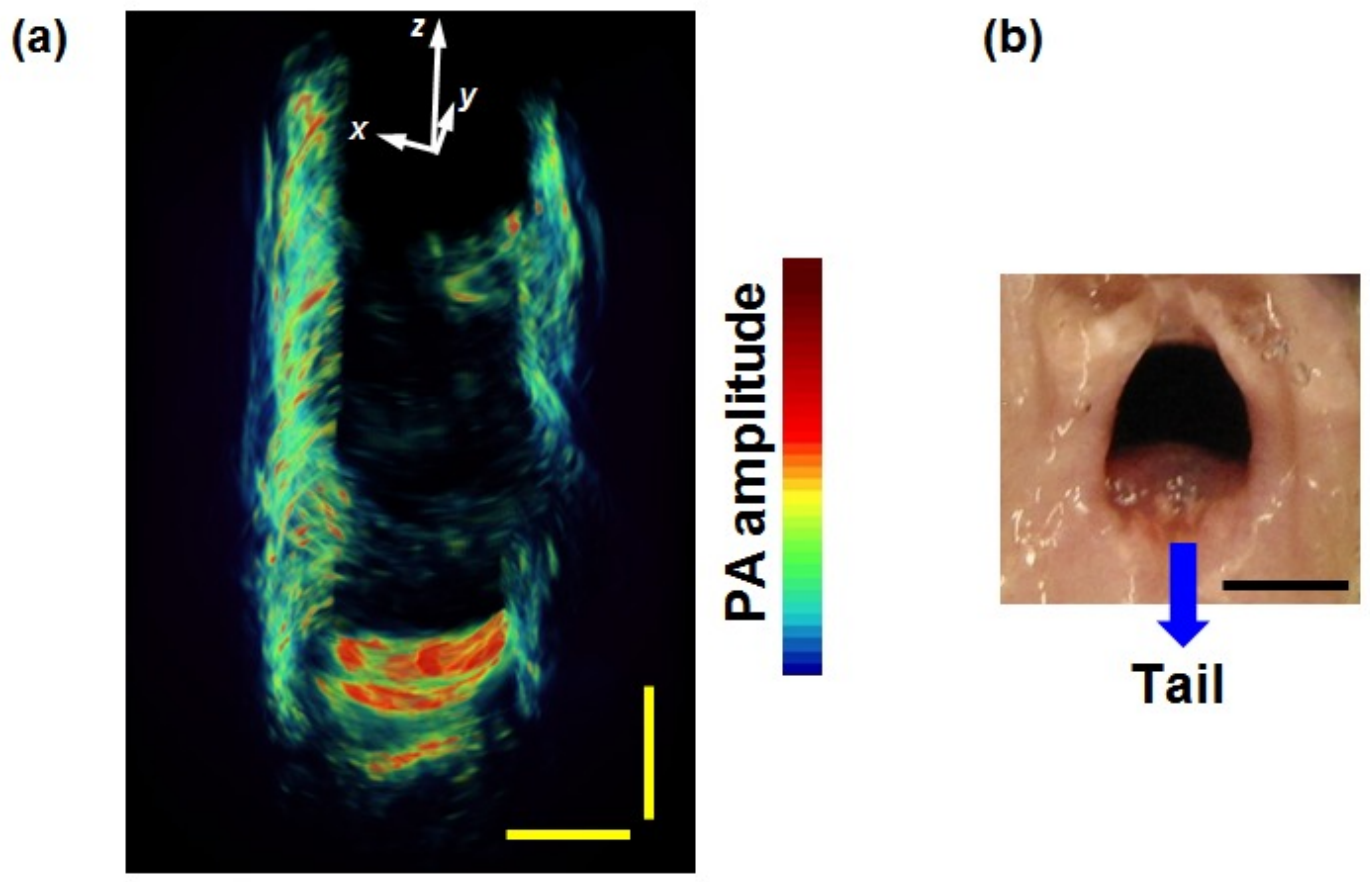

Figure 3. (a) Three-dimensionally rendered PA structural image obtained with a $578 \mathrm{~nm}$ laser excitation wavelength. The down side of this image is closer to anus, and the negative $y$-axis corresponds to the dorsal direction of the animal, respectively. (b) A photo showing the melanoma tumor in the rat colorectum. The scale bars represent $5 \mathrm{~mm}$.

In this study, we investigated PA image features of melanoma tumor in the colorectum of a nude rat in situ to show PAE's clinical potential. Many types of information related to tumor, such as its site, size, developing rate, vascular structure and growth pattern would be important, because they could be used for evaluating degree of maturity, distinguishing adenomas and carcinomas, and determining a prognosis. As we presented here, PAE was able to provide information on the tumor location and adjacent blood vasculature clearly without using any contrast agent. The experimental results show PAE's potential to be used as a new clinical tool in diagnosing many gastrointestinal diseases, such as cancer. 
For future studies, we plan to use multi-colored laser light to noninvasively provide clinically relevant information like total hemoglobin concentration, blood oxygenation, blood flow, and temperature, and we will also try to perform a colorectal tumor imaging study using nude rats.

\section{CONCLUSION}

We performed the first in vivo PA endoscopic imaging study of melanoma tumor growth in the colorectum of a nude rat. PAE could provide the melanoma tumor region and its adjacent blood vasculature clearly without using any contrast agent. Our study could lead to a useful methodology for studying tumor development in small animals.

\section{ACKNOWLEDGEMENT}

We thank Seema Dahlheimer for her attentive reading of the manuscript. This work was sponsored in part by National Institutes of Health grants R01 CA157277, R01 NS46214 (BRP), R01 EB000712, R01 EB008085, and U54 CA136398 (Network for Translational Research). L.W. has a financial interest in Microphotoacoustics, Inc. and Endra, Inc., which, however, did not support this work.

\section{REFERENCES}

1. J. M. Yang, K. Maslov, H. C. Yang, Q. Zhou, K. K. Shung and L. V. Wang, "Photoacoustic endoscopy," Opt Lett 34(10), 1591-1593 (2009)

2. J. M. Yang, C. Favazza, R. Chen, J. Yao, K. Maslov, X. Cai, Q. Zhou, K. K. Shung and L. V. Wang, "Volumetric photoacoustic endoscopy of upper gastrointestinal tract: ultrasonic transducer technology development " Proc. SPIE 7899, 78990D (2011)

3. J. M. Yang, C. Favazza, R. Chen, J. Yao, X. Cai, K. Maslov, Q. Zhou, K. K. Shung and L. V. Wang, "Simultaneous functional photoacoustic and ultrasonic endoscopy of internal organs in vivo," Nat Med 18(8), 1297-1302 (2012)

4. J. M. Yang, R. Chen, C. Favazza, J. Yao, C. Li, Z. Hu, Q. Zhou, K. K. Shung and L. V. Wang, "A 2.5-mm diameter probe for photoacoustic and ultrasonic endoscopy," Opt Express 20(21), 23944-23953 (2012)

5. L. V. Wang, "Multiscale photoacoustic microscopy and computed tomography," Nat Photonics 3(9), 503-509 (2009)

6. L. V. Wang and S. Hu, "Photoacoustic tomography: in vivo imaging from organelles to organs," Science 335(6075), 1458-1462 (2012)

7. J. Yao, K. I. Maslov, Y. Shi, L. A. Taber and L. V. Wang, "In vivo photoacoustic imaging of transverse blood flow by using Doppler broadening of bandwidth," Opt Lett 35(9), 1419-1421 (2010)

8. J. Yao, K. I. Maslov, Y. Zhang, Y. Xia and L. V. Wang, "Label-free oxygen-metabolic photoacoustic microscopy in vivo," J Biomed Opt 16(7), 076003 (2011)

9. J. Yao, C. H. Huang, L. Wang, J. M. Yang, L. Gao, K. I. Maslov, J. Zou and L. V. Wang, "Wide-field fast-scanning photoacoustic microscopy based on a water-immersible MEMS scanning mirror," J Biomed Opt 17(8), 080505080501 (2012)

10.L. Gao, L. Wang, C. Li, Y. Liu, H. Ke, C. Zhang and L. V. Wang, "Single-cell photoacoustic thermometry," J Biomed Opt 18(2), 26003 (2013)

11. Y. Wang, K. Maslov and L. V. Wang, "Spectrally encoded photoacoustic microscopy using a digital mirror device," J Biomed Opt 17(6), 066020 (2012)

12. M. R. Chatni, J. Yao, A. Danielli, C. P. Favazza, K. I. Maslov and L. V. Wang, "Functional photoacoustic microscopy of pH," J Biomed Opt 16(10), 100503 (2011)

13.Z. Guo, S. Hu and L. V. Wang, "Calibration-free absolute quantification of optical absorption coefficients using acoustic spectra in 3D photoacoustic microscopy of biological tissue," Opt Lett 35(12), 2067-2069 (2010)

14.C. Zhang, K. Maslov and L. V. Wang, "Subwavelength-resolution label-free photoacoustic microscopy of optical absorption in vivo," Opt Lett 35(19), 3195-3197 (2010)

15.D. K. Yao, K. Maslov, K. K. Shung, Q. Zhou and L. V. Wang, "In vivo label-free photoacoustic microscopy of cell nuclei by excitation of DNA and RNA," Opt Lett 35(24), 4139-4141 (2010) 
16. M. Pramanik and L. V. Wang, "Thermoacoustic and photoacoustic sensing of temperature," J Biomed Opt 14(5), 054024 (2009)

17.J. T. Oh, M. L. Li, H. F. Zhang, K. Maslov, G. Stoica and L. V. Wang, "Three-dimensional imaging of skin melanoma in vivo by dual-wavelength photoacoustic microscopy," J Biomed Opt 11(3), 34032 (2006)

18. H. F. Zhang, K. Maslov, G. Stoica and L. V. Wang, "Functional photoacoustic microscopy for high-resolution and noninvasive in vivo imaging," Nat Biotechnol 24(7), 848-851 (2006)

19.G. S. Filonov, A. Krumholz, J. Xia, J. Yao, L. V. Wang and V. V. Verkhusha, "Deep-tissue photoacoustic tomography of a genetically encoded near-infrared fluorescent probe," Angew Chem Int Ed Engl 51(6), 1448-1451 (2012)

20. J. Laufer, P. Johnson, E. Zhang, B. Treeby, B. Cox, B. Pedley and P. Beard, "In vivo preclinical photoacoustic imaging of tumor vasculature development and therapy," J Biomed Opt 17(5), 056016 (2012)

21.E. C. Cho, Y. Zhang, X. Cai, C. M. Moran, L. V. Wang and Y. Xia, "Quantitative analysis of the fate of gold nanocages in vitro and in vivo after uptake by U87-MG tumor cells," Angew Chem Int Ed Engl 52(4), 1152-1155 (2013) 